\title{
The Affect of Variable Pressure on the Quality of Energy Dispersive X-ray Analysis Results
}

\section{P. DeVries}

The Boeing Company, M/C/ W-S25-C563, 2020 E. Imperial Highway, E1 Segundo, CA 90245 USA

Scanning electron microscopy has been an extremely useful addition to the analyst's tool box due to its ability to produce high resolution images with large depth of field at very high magnifications. By adding energy dispersive $x$-ray spectroscopy (EDS), the microscopist can extend his or her investigation into the chemical composition of the material under study. Both of these tools have proven themselves invaluable when addressing the issue of Foreign Object Debris (FOD).

Chemical characterization of FOD using EDS analysis works relatively well when the unknown material is electrically conductive and the debris is presented on a conductive media or substrate. Unfortunately the debris and the mounting media on which FOD is presented are often non-conductive (Figure 1) resulting in sample charging. Sputter coating can resolve the charging issue, however the thick coating required to render an electrically insulative tape or the rough texture of the cloth conductive can significantly interfere with debris analysis. The use of variable pressure (VP) reduces charging of these samples to a point where quality imaging can be performed without sputter coating (Figure 2) however, as seen in Table 1, EDS results may vary notably particularly with respect to carbon and oxygen depending on the pressure used during the evaluation (Table 1). Subsequent normalization of EDS data then automatically shifts the values of the remaining elements. Identifying this erroneous compositional data can be difficult even when one is aware of the problem. The use of EDS under variable pressure is discussed in detail by Newbury ${ }^{1}$

This paper will take an operators view into the extent and affects of beam scatter (skirting) and sample charging on the veracity of EDS results when performing FOD analysis on non-conductive tape and fabric substrates under variable pressure conditions up to 1 Torr. A review of methods for identifying erroneous data and minimizing deleterious effects of variable pressure on the analysis will be discussed.

[1] D Newbury; Journal of Research of the National Institute of Standards and Technology 107, (2002), p. 567-603 


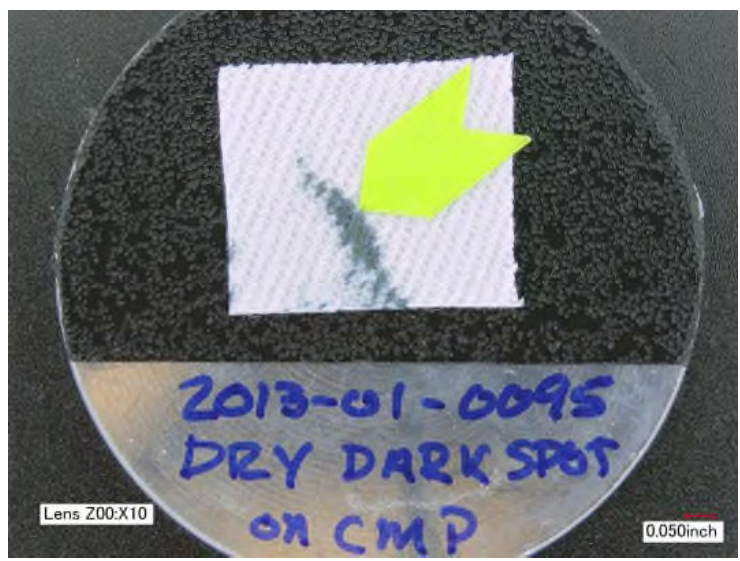

Figure 1 - The black debris on twill jean cloth is typical of contamination samples submitted for energy dispersive $\mathrm{x}$-ray spectroscopy (EDS). .
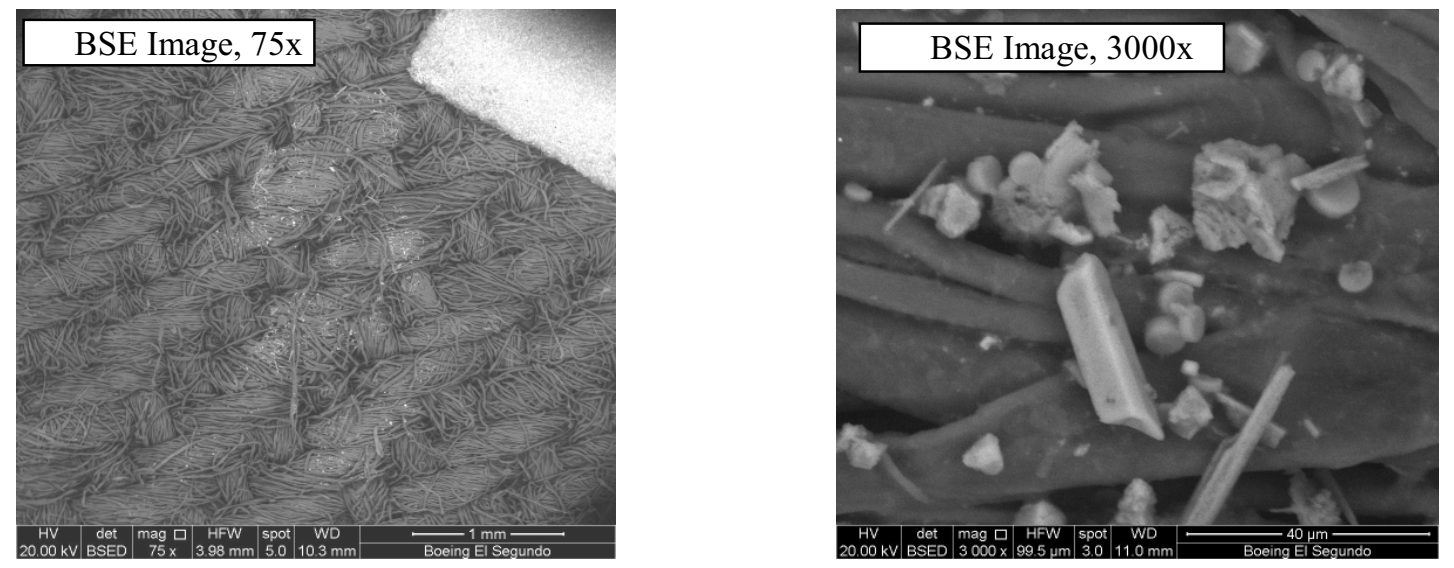

Figure 2: Operating in variable pressure mode (0.98 Torr) addresses charging of the twill jean cloth and allows quality images to be obtained of individual particulates.

Table 1: EDS Results, FOD Particles

\begin{tabular}{|c|c|c|c|c|c|c|c|c|}
\hline Location & Mode & $\mathbf{C}$ & $\mathbf{O}$ & $\mathbf{F}$ & $\mathbf{N a}$ & $\mathbf{M g}$ & $\mathbf{S i}$ & $\mathbf{S}$ \\
\hline \hline \multirow{2}{*}{ Particle 1 } & PP & 45.50 & 42.72 & 6.56 & 1.04 & 0.21 & 0.93 & 0.33 \\
\cline { 2 - 9 } & Hvac & 64.41 & 31.09 & 1.30 & 0.54 & & 1.74 & \\
\hline \multirow{2}{*}{ Particle 2 } & PP & 44.78 & 41.07 & 6.98 & 0.85 & 0.28 & 1.02 & 0.64 \\
\cline { 2 - 9 } & Hvac & 46.90 & 34.29 & 4.41 & & & 1.93 & 1.24 \\
\hline \multirow{2}{*}{ Particle 3 } & PP & 34.86 & 42.76 & 8.61 & 1.03 & 3.44 & 1.14 & 4.60 \\
\cline { 2 - 9 } & Hvac & 22.35 & 51.48 & 4.00 & 0.89 & 7.93 & 0.65 & 8.95 \\
\hline
\end{tabular}

EDS performed on particles under partial pressure (PP / 0.98 Torr) and full vacuum (HVac) produce a significant shift in carbon and oxygen results due to beam "skirting" and atmospheric contributions which automatically shift the remaining elements when the analysis is normalized (Not all elements shown). 